Ann. Biol. anim. Bioch. Biophys., $\mathbf{1}_{96}, 5$ (2), 249-265.

\title{
LIEU D'ABSORPTION DES GRAISSES DANS L'INTESTIN GRÊLE DU PORC
}

\author{
N. VOdOVAR, Claudine THIEULIN, Andrée PIHET, J. FLANZY \\ Station centrale de Nutrition, \\ Centre national de Recherches zootechniques, Jouy-en-Josas (Seine-et-Oise)
}

SOMMAIRE

Trois séries de porcs ont reçu un repas équilibré en protides et glucides, mais surchargé en matières grasses (triglycérides naturels, triglycérides homogènes ou acides gras libres, suivant le lot), afin que, sur tout le parcours du nutriment entre le pylore et la valvule de Bauhin, il reste, dans le contenu intestinal, une quantité suffisante de graisse pour que l'absorption puisse avoir lieu.

Les animaux ont été abattus $6 \mathrm{~h}$ 30 après l'ingestion de l'aliment, moment déterminé comme étant celui où l'intestin grêle, sur toute sa longueur, est impliqué dans le processus d'absorption.

L'observation au microscope optique de coupes de paroi intestinale prélevées sur Io segments pris à des niveaux différents a permis de constater que les I 6 matières grasses expérimentées commencent à être absorbées au début du duodénum et que leur absorption dans les conditions de l'expérience se poursuit jusqu'à $30 \mathrm{~cm}$ environ avant la valvule de Bauhin. L'intensité apparente de l'absorption diffère suivant la nature des graisses ; si on observe que l'intensité d'absorption varie également suivant les segments, ceci peut être expliqué par le fait que la vitesse de transit, l'état physico-chimique et la concentration des graisses sont différents suivant le segment considéré.

En conclusion, on peut dire que, dans les conditions de nos expériences, il n'y a pas de lieu électif suivant leur nature pour l'absorption des différentes matières grasses, mais que celles-ci sont absorbées tout le long de l'intestin grêle.

\section{I. -- INTRODUCTION}

Comme Razumov ( 1963 ) l'a démontré, bien que le tractus digestif soit impliqué, en entier, dans le processus de transformation et de transport des graisses après leur ingestion, les cellules épithéliales absorbantes de l'intestin grêle restent le facteur principal de l'absorption. Étudiées au microscope optique, et si on tient compte de leur emplacement sur les villosités et de l'âge de l'animal, ces cellules sont, chez le Porc, sur toute la longueur de l'intestin grêle, morphologiquement identiques (VoDo- 
VAR, I964). Il en est de même pour leur ultrastructure étudiée au microscope électronique (VODOVAR, FLÉCHON, I964). Compte tenu de ces constatations, il reste à savoir si les différentes graisses peuvent être absorbées par la muqueuse tout le long de l'intestin grêle et, dans l'affirmative, si le lieu d'absorption ne dépendra que de leur état physico-chimique, de leur vitesse de transit et de leur concentration, ou bien si les différents segments de 1'intestin les absorbent exclusivement ou préférentiellement suivant leur nature, ce qui permettrait de penser que la cytochimie de la couche épithéliale est différente suivant les segments.

Par l'étude histologique de coupes de la paroi intestinale du Rat, après ingestion d'huile d'olive ou d'acide oléique, FrazER (I943) retrouve les deux substances dans la muqueuse du jéjunum proximal. Pour BERNHARd, WAGNer et Ritzer (I952), c'est également dans la partie proximale du jéjunum que les graisses quittent la lumière intestinale. Les analyses du contenu intestinal, prélevé chez l'Homme, à différents niveaux, par tubage, après ingestion d'un repas équilibré d'huile de maïs, de poudre de lait, de dextrose et contenant une substance de référence soluble, non absorbable, ont permis à BoRgström et al. (I957), à BoRGSTRöM, DAHLqVisT et LUND (I962) de conclure que l'absorption des graisses se fait dans la partie distale du duodénum et les cent premiers centimètres du jéjunum. KNOEBEL et NASSET (I957) ont constaté que, chez les chiens porteurs d'une fistule de la partie proximale du jéjunum, un repas d'huile de coton et de mono-linoléine était absorbé avant la fistule dans une proportion allant jusqu'à $70 \mathrm{p}$. Ioo des graisses administrées. Pour Johnston (I959), l'absorption, in vitro, de l'acide palmitique ${ }^{14} \mathrm{C}$, chez le Hamster, se fait également au début du jéjunum.

Ces travaux, qui semblent démontrer que les graisses de nature différente sont absorbées par la partie proximale de l'intestin grêle, sont controversés: certains auteurs, en effet, pensent que c'est la partie distale de 1'intestin qui absorbe, de préférence, les graisses.

En pratiquant la résection de différentes parties de l'intestin, chez le Chien, KREMEN, LINNER et NELSON (I954) observent que l'absorption des graisses est moins complète quand la partie distale a été éliminée, ce qui rejoint la conclusion antérieure de Jensenius (I945). Pour Benson et al. (I956), Chandlek et Benson (I956), l'huile d'olive marquée à $1^{\prime 131} I$ et ingérée par des rats, est retrouvée dans la partie distale de l'intestin. Des résultats semblables ont été obtenus par ABERDEEN, SHEPHERD et Simmonds (I96o), puis par BennetT et Srmmonds (I962), avec 1'huile de coprah administrée à des rats non anesthésiés, à l'aide d'une sonde. Ces résultats concordent avec les observations histologiques de McNAUGHT (I959).

Dans un troisième groupe de travaux, on peut classer les expériences qui tendent à démontrer que le lieu d'absorption dépend surtout de la nature de la substance ingérée.

Ainsi, sur des chiens nourris avec de la viande maigre, à laquelle on a incorporé de la triélaïdine, FAVARGER (I949) constate la présence de phospholipides dans les parois du duodénum, mais il trouve les glycérides et les acides gras dans les parois de l'iléon.

A la suite de ses expériences, Turner (I958, $a$ et $b$ ) conclut que, chez l'Homme et le Chien, la trioléine est absorbée par le duodénum et le jéjunum, tandis que l'acide oléique est absorbé dans la dernière partie de l'intestin grêle. Les résultats de GRENIER et al. (I962, I963) s'opposent aux précédents, bien que les expériences aient 
été pratiquées sur la même espèce animale : l'acide oléique est absorbé dans le jéjunum proximal et la trioléine dans l'iléon.

D'autres expériences montrent que le lieu d'absorption de différentes substances grasses dépend également du mélange dans lequel elles ont été ingérées.

FAVARGER et GERLACH (I953), en faisant ingérer différentes graisses et différents acides gras marqués, constatent que, outre que l'élaïdine disparaît dans les premier et deuxième tiers et la stéarine dans la partie distale de l'intestin, les acides gras libres, ajoutés à des graisses, sont absorbés plus rapidement que dans le cas où ils ne sont pas mélangés avec des triglycérides. Ceci rejoint l'expérience de REISER (I950), qui comparait l'absorption de l'acide linoléique seul à celle de l'acide linoléique fixé sur un triglycéride; celle de BORGSTRöM (I952), qui constate que les acides gras libres présents dans les graisses apparaissent dans la lymphe plus rapidement que les graisses neutres, mais que, ingérés séparément, ils apparaissent bien plus tardivement que les triglycérides correspondants. Il en est ainsi pour 1'acide oléique et l'huile d'olive (MALM, REEMTSMA et BARKER, I956). Tous ces auteurs concluent à une absorption moindre et plus lente de l'acide libre.

Les résultats, tout récents, de BENNETT (I964) montrent que, chez le Rat, les acides gras à longueurs de chaînes différentes sont absorbés indifféremment par n'importe quel segment du jéjunum-iléon.

Comme on vient de le voir, la plupart des expériences concernant le lieu d'absorption des graisses ont été faites sur le Rat, quelquefois sur le Chien, rarement sur l'Homme, avec des méthodes différentes, souvent dans des conditions physiologiques susceptibles de provoquer des perturbations complètes ou partielles de l'absorption. Les résultats obtenus sont rarement concordants, souvent contradictoires. Dans aucun des cas, il n'est possible de savoir quels sont les facteurs déterminants dans le processus d'absorption des matières grasses ingérées.

La solution de ce problème présente un intérêt évident, tant dans le domaine de la recherche biochimique que dans celui de la médecine. C'est ce qui nous a amené à faire une série d'expériences sur un vertébré supérieur monogastrique tel que le Porc.

\section{II. - MATÉRIEI.}

Les animaux sacrifiés pour ces expériences étaient des porcs en croissance, de poids vif compris entre 50 et $60 \mathrm{~kg}$, de race Large White, en bon état sanitaire et au développement normal.

On a utilisé les corps gras suivants : des triglycérides naturels (huile de coprah, huile d'olive, huile de coton, huile de maïs et saindoux), des triglycérides homogènes (triburyrine, trilaurine, tripalmitine, tristéarine et trioléine), ainsi que des acides gras (acide laurique, acide palmitique, acide stéarique, acide oléique et acide linoléique), les produits fabriqués ayant été fournis par les laboratoires Rhône-Poulenc, et la Nutritional Biochemical Corporation

Au moyen de coupes histologiques, on étudie le lieu et l'intensité apparente de pénétration de différents lipides naturels, de triglycérides homogènes et de différents acides gras dans la muqueuse intestinale du Porc, après ingestion d'un repas auquel, à chaque fois, il a été incorporé une des graisses à étudier.

\section{A. Essais préliminaires}

Avant d'aborder la partie expérimentale proprement dite, il a été nécessaire d'établir :

$1^{0}$ La durée du transit intestinal nécessaire pour que la première fraction du repas ingéré parvienne à la valvule de Bauhin, de façon que l'intestin grêle des animaux, au moment de l'abattage, soit impliqué sur toute sa longueur dans les processus de digestion et d'absorption, avant que l'estomac ne soit entièrement vidé. Ce temps est d'environ 6 heures, pour le repas choisi, mais pour 
échapper aux variations individuelles, les abattages ont été faits $6 \mathrm{~h}$ zo après l'ingestion.

$2^{\circ}$ La quantité de graisse à incorporer au repas pour que, à tous moments et sur toute la londe l'intestin grêle, existe dans le bol alimentaire une surcharge suffisante de cette substance, afin que la cellule épithéliale absorbante puisse exercer sa fonction d'absorption au niveau de tous les segments où elle est apte à le faite. Cette quantité a été fixée uniformément à $100 \mathrm{~g}$ : elle est suffisante pour toutes les matières expérimentées, sans être toujours nécessaire, exception faite pour la tributyrine, pour laquelle Ia capacité absorbante de l'intestin grêle s'est révélée supérieure.

Pour ces essais préliminaires, des animaux de même poids vif $(50$ à $60 \mathrm{~kg}$ ) ont été sacrifiés à intervalles réguliers, après ingestion d'un repas-type de $500 \mathrm{~g}$, équilibré en protéines et en glucides mais surchargé en graisses.

\section{B. - Expérimentation}

Les porcs ont été soumis à un régime sans lipides, durant 48 heures; puis, sans que leur nutrition en soit pour autant perturbée, au repas précédant l'abattage, il a été incorporé ioo $\mathrm{g}$ soit d'un triglycéride naturel, soit d'un triglycéride homogène, soit d'un acide libre (les expériences n'ont pas été faites avec des mélanges). $6 \mathrm{~h}$ 30 après l'ingestion, les animaux ont été sacrifiés : abattages, prélèvements et fixations ont été pratiqués suivant les techniques décrites précédemment (Vodovar, I964).

Les prélèvements de courts segments de 5 à $10 \mathrm{~cm}$ d'intestin ont d'abord été effectués au niveau du pylore, puis à l'angle duodéno-jéjuno-iléal (tabl. r, 2, 3, A et B), près de la valvule de Bauhin et à $30 \mathrm{~cm}$ avant celle-ci (tabl. I, 2, 3, I et J), enfin sur le reste du jéjunum-iléon, soit environ sur I I mètres et respectivement à $\mathrm{I}, 50 \mathrm{~m}, 3 \mathrm{~m}, 4,50 \mathrm{~m}, 6 \mathrm{~m}, 7,50 \mathrm{~m}$ et $9 \mathrm{~m}$ après l'angle duodéno-jéjunoiléal (tabl. $1,2,3$, C à H).

L'intensité apparente d'absorption, évaluée en fonction de la graisse trouvée dans la cellule épithéliale absorbante, est désignée par $x=$ très faible, $2 x=$ faible, $3 x=$ moyenne, $4 x=$ forte, $5 x=$ très forte, cette intensité est mesurée par cytophotomètre et les valeurs comparatives sont seules retenues. L'intestin grêle, entre l'angle duodéno-jujéno-iléal et la valvule de Bauhin, est désigné sous le vocable de jéjunum-iléon, faute d'avoir pu établir une limite anatomique valable entre ce qu'on désigne souvent par jéjunum et par iléon. Les tissus ont été coupés à $6 \mu$ par congélation au cryostat International Equipement Company, soit sans fixation, soit après fixation de 6 à 12 heures dans le formol-calcium. La coloration des coupes a été pratiquée au Noir Soudan B et carmin aluné, au Rouge Soudan III et hématoxyline, soit à la température du laboratoire, soit à la température de fusion de la graisse, et au Bleu de Nil ; le montage a été fait dans le sirop d'Apathy.

Avant d'aborder les résultats de nos expériences, nous pensons qu'il est nécessaire de souligner que les méthodes histochimiques, dont nous avons fait choix, ne sont pas à l'abri des critiques, comme la plupart des méthodes d'ailleurs. Ainsi, à l'heure actuelle les déterminations quantitatives d'une substance chimique, malgré les progrès de la cytophotométrie, ne nous semblent pas toujours satisfaisantes. C'est pourquoi nous nous sommes borné à ne retenir que les valeurs comparatives. Cependant, si ces valeurs peuvent être considérées comme très satisfaisantes quand il s'agit de mesurer l'intensité d'absorption de mêmes graisses sur des coupes identiques, elles le sont moins quand il s'agit de graisses de nature différente et dont les colorations peuvent varier. L'avantage de la méthode histochimique choisie est surtout de pouvoir affirmer sans contestation possible, quelles sont les parties de l'intestin où l'absorption se fait réellement et de déterminer à quel moment elle se fait.

\section{IV. - OBSERVATIONS E'T RÉSULTATS}

Les observations ont porté sur 1'intestin de 3 lots de 7 porcs, chacun ayant ingéré un repas surchargé, le premier en triglycérides naturels, le deuxième en triglycérides homogènes et le troisième en acides gras libres.

A l'abattage des animaux, il a été vérifié que l'intestin grêle, sur toute sa longueur, était toujours impliqué dans les processus d'absorption et qu'en tous les points, il restait dans le contenu intestinal une quantité suffisante de graisse ingérée, pour que l'absorption des graisses ait pu avoir lieu.

L'examen histologique des coupes de tissu de l'intestin grêle du Porc, prélevé après ingestion de graisses, à différents niveaux compris entre le pylore et la valvule 


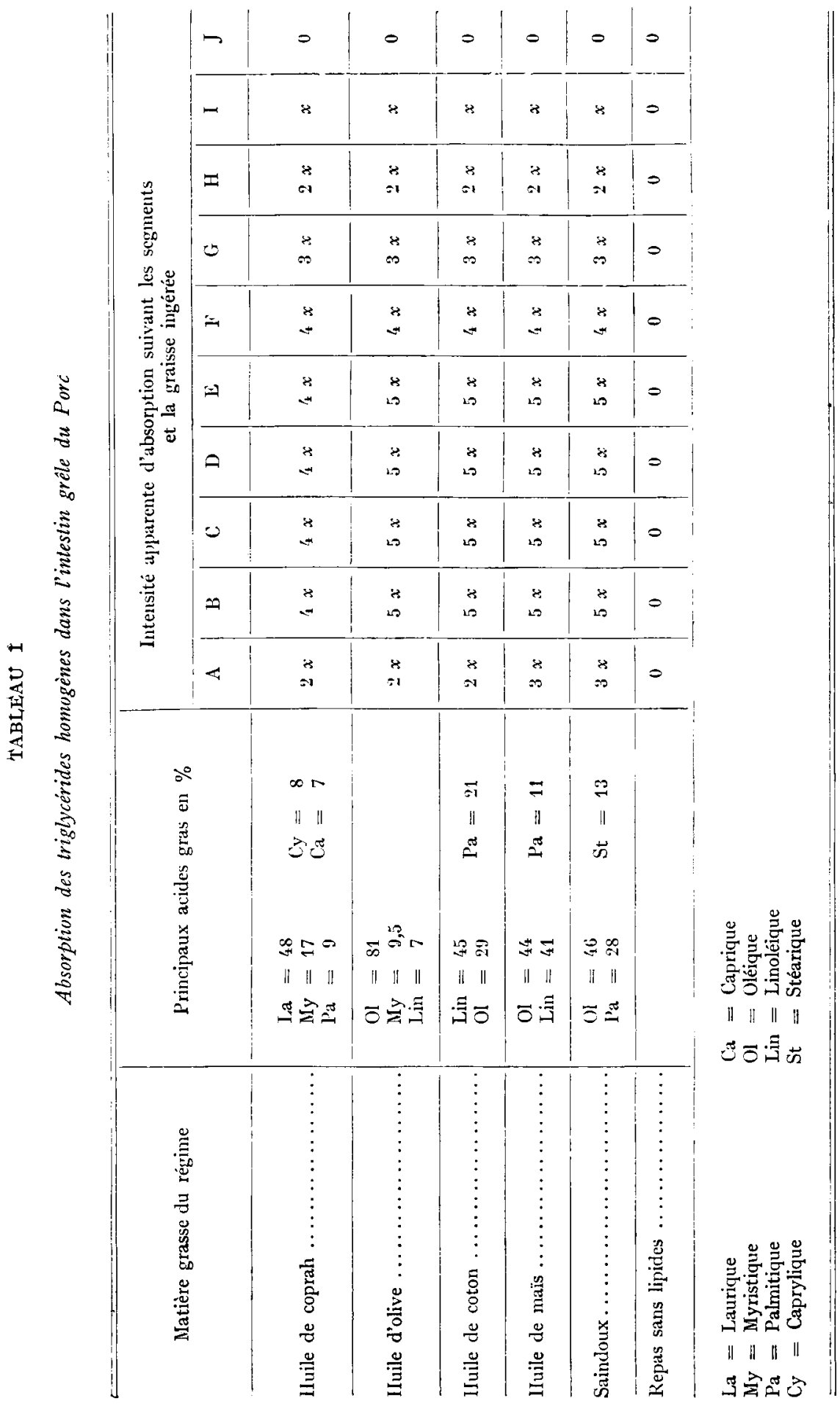


de Bauhin, permet de situer indiscutablement, dans les conditions d'expérience, le lieu d'absorption de ces différentes graisses. Il permet également de comparer l'intensité d'absorption apparente, suivant les segments, pour la même graisse, et entre des segments correspondants pour des graisses de nature différente; il permet d'apprécier également la surface absorbante, ainsi que l'aspect et la grandeur des particules dans l'espace intermicrovilleux et à l'intérieur de la cellule épithéliale absorbante.

\section{A. Triglycérides naturels}

L'absorption des triglycérides naturels expérimentés, (non purifiés), (tab1. I), commence, invariablement, dès les premiers centimètres après le pylore. Le début de l'absorption coïncide avec le débouché du canal cholédoque dans le duodénum, qui se situe, chez le Porc, entre le $2^{\mathrm{e}}$ et le $6^{\mathrm{e}}$ centimètre après le pylore (Vodovar, FLANZy et François, I964) et, au minimum, I2 $\mathrm{cm}$ avant le débouché du canal pancréatique (les animaux sacrifiés n'en possédaient qu'un). Elle se poursuit sur toute la longueur du duodénum et du jéjunum-iléon jusqu'à environ $30 \mathrm{~cm}$ avant la valvule de Bauhin.

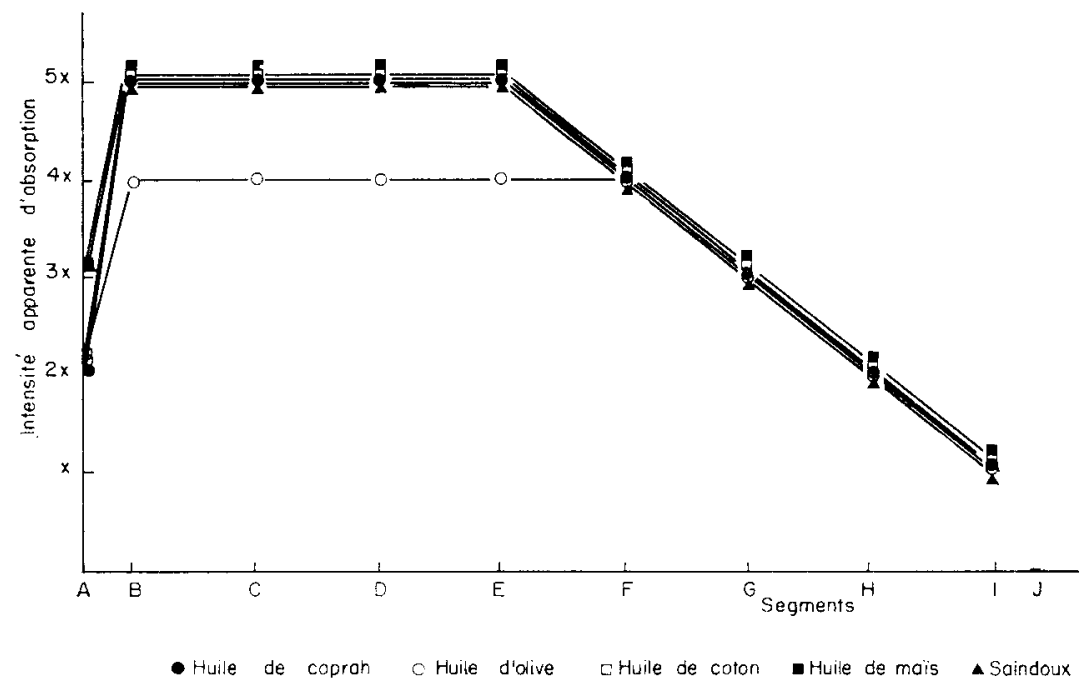

FIo. I. - Absorption des triglycérides naturels dans l'intestin grêle du Porc

En comparant l'intensité d'absorption apparente de chaque triglycéride, suivant les segments (tabl. I), on constate qu'au début du duodénum, où l'absorption commence, l'intensité de celle-ci est toujours faible; la surface absorbante, à ce niveau, est située seulement au sommet des villosités, quels que soient les triglycérides.

Dans la partie distale du duodénum et sur les deux tiers du jéjunum-iléon environ, l'absorption est d'une intensité relativement uniforme et maximum par rapport aux autres segments (fig. I) quelle que soit la nature des triglycérides. Ia surface absorbante est variable suivant les segments: elle est importante surtout vers la fin du duodénum et sur le premier tiers du jujénum-iléon. Dans cette partie de l'intestin, l'absorption se fait, en moyenne, sur les deux tiers distaux de la villosité. 


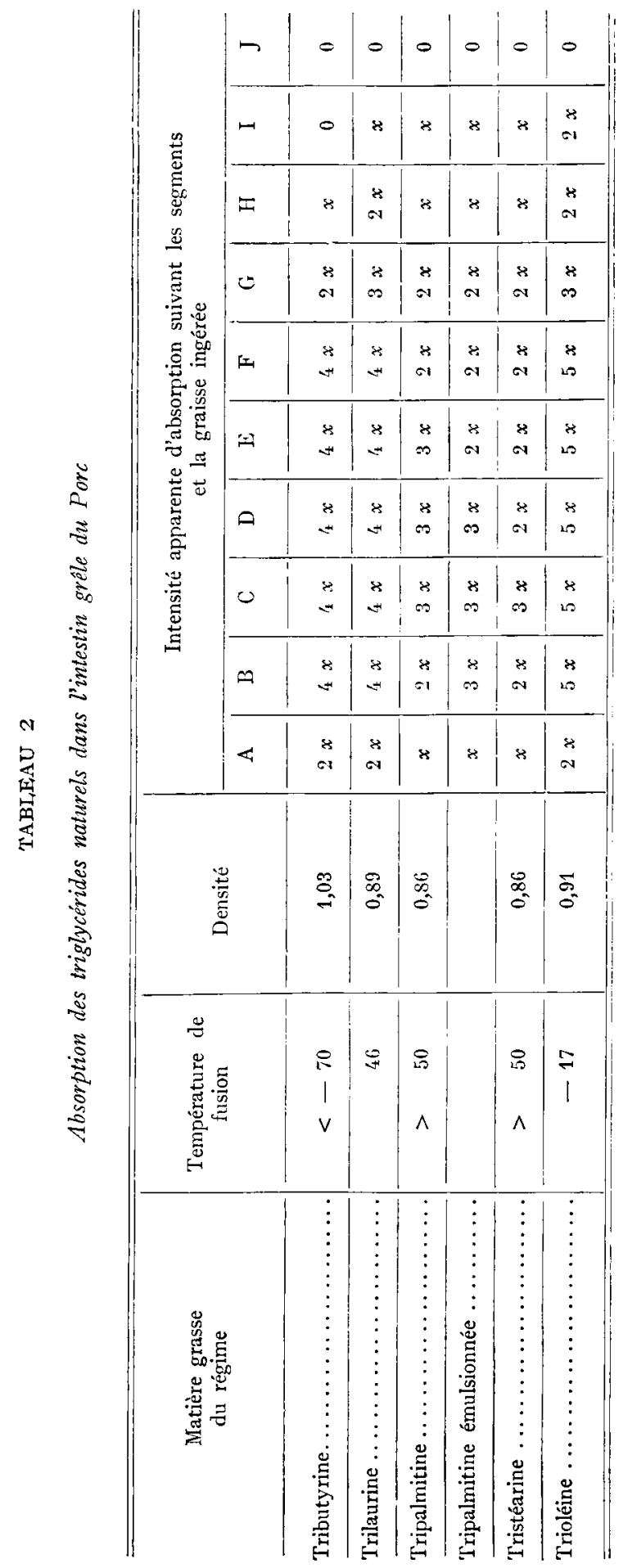


Dans la troisième partie du jéjunum-iléon, l'intensité de l'absorption et la surface absorbante diminuent au fur et à mesure qu'on se rapproche de la valvule de Bauhin.

Si la comparaison de l'intensité d'absorption d'un triglycéride sur les différents segments de l'intestin est relativement aisée, celle de triglycérides différents sur les segments homologues d'intestin est toujours moins précise. Ceci est dû à la densité et aux dimensions des particules propres à chaque triglycéride, d'une part, à la différence de coloration des différentes graisses, d'autre part. Il semble, toutefois que le saindoux et l'huile de maïs soient absorbés dans la première partie du duodénum avec plus d'intensité que l'huile d'olive, l'huile de coton et l'huile de coprah.

Dans le reste de l'intestin, c'est surtout avec l'huile de coprah que l'intensité d'absorption apparente est plus faible.

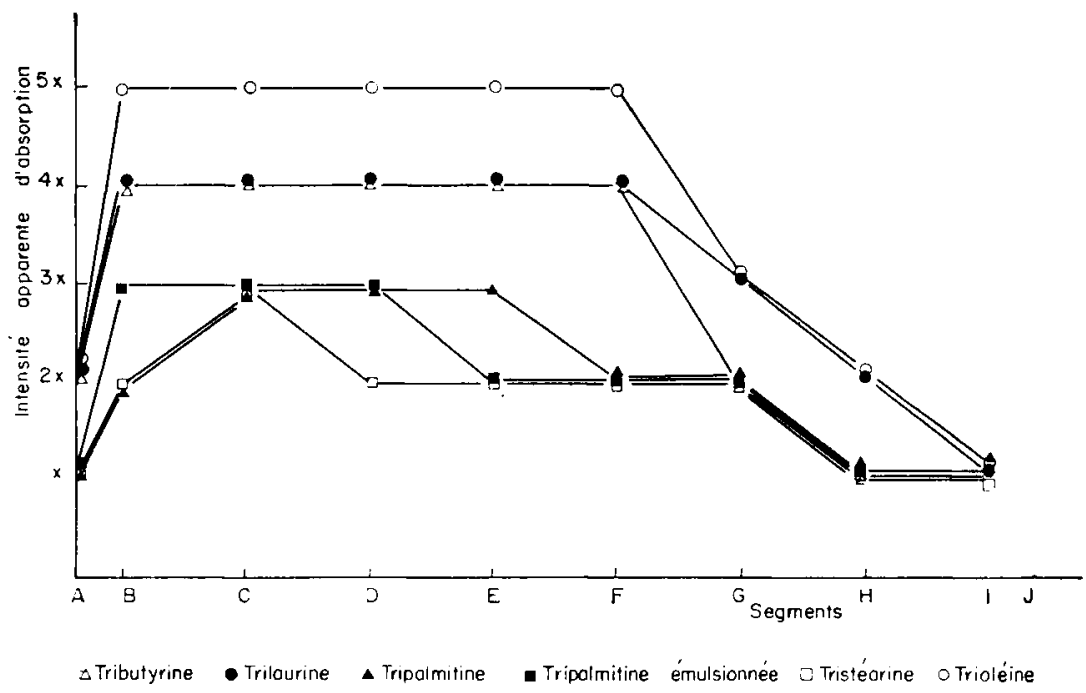

Fig. 2. - Absorption des triglvcérides homogènes dans l'intestin grèle du Porc

On remarque que les dimensions des particules de tous les glycérides expéri. mentés, parvenues dans les espaces intermicrovilleux, sont relativement uniformes: mesuré à l'oculaire micrométrique, leur diamètre est égal ou inférieur à $0,3 \mu$. C'est pour le saindoux qu'il est le plus grand, pour l'huile de maîs le plus petit.

L'aspect des particules (qui ont la forme de gouttelettes, sauf celles d'huile de maïs qui sont moins caractéristiques) et leur grandeur à l'intérieur de la cellule absorbante (fig. 4, 5) sont particuliers à chaque triglycéride.

\section{B. Triglycérides homogènes}

Comme pour les triglycérides naturels, l'absorption des triglycérides homogènes expérimentés (tab1. 2) commence immédiatement après le pylore et se poursuit, sans discontinuité, tout le long de l'intestin grêle, excepté sur une courte portion précédant la valvule de Bauhin.

La tributyrine, elle, ne se trouve plus dans la paroi de l'intestin à partir du 
$9^{\mathbf{e}}$ mètre. A ce niveau, la quantité qu'on en retrouve dans le contenu intestinal est très faible, ce qui confirme que l'absorption en a été plus rapide que celle des autres triglycérides.

L'intensité d'absorption apparente des triglycérides homogènes, suivant les segments de l'intestin (fig. 2) est comparable ou inférieure à celle des triglycérides naturels.

Dans la partie proximale du duodénum, l'intensité est très faible pour la tripalmitine et la tristéarine, faible pour la tributyrine, la trilaurine et la trioléine. C'est dans le reste du duodénum et les deux premiers tiers du jéjunum-iléon que l'intensité d'absorption apparente est la plus élevée ; sur le troisième tiers; elle diminue progressivement pour l'ensemble des triglycérides expérimentés dans cette série.

Pour tous, la surface absorbante, au début du duodénum, se limite au sommet des villosités; pour le tiers distal du duodénum et le tiers proximal du jéjunum-iléon, les deux tiers - approximativement - des cellules épithéliales des villosités contiennent des substances soudanophiles, quoique les surfaces absorbantes diffèrent suivant les triglycérides (elle sont plus importantes pour la tributyrine et la trioléine). Pour le reste de l'intestin, la surface d'absorption est assez variable.

Si on compare l'intensité apparente d'absorption des différents triglycérides homogènes sur des segments homologues, on constate (tab1. 2 et fig. 2) que la différence est très marquée entre celle de la trioéline, qui est la plus élevée, et celle de la tributyrine et de la trilaurine, d'une part, entre celle de la tripalmitine et celle de la tristéarine, d'autre part, et que, pour la tripalmitine émulsionnée avant ingestion, elle est, sur quelques segments, légèrement supérieure à celle de la tripalmitine non émulsionnée.

L'aspect de la substance soudanophile, dans les espaces intermicrovilleux et intercellulaires (fig. 6,7), diffère suivant les triglycérides. L'homogénéité des particules dans l'espace intermicrovilleux pour chacun des triglycérides est très caractéristique, ce qui n'est pas le cas pour celles qui se trouvent à l'intérieur des cellules.

On peut remarquer que l'absorption de la tributyrine est bien plus rapide que celle des autres triglycérides homogènes. Ses particules, dont le diamètre est de $0,2 \mu$ environ, sont toujours homogènes, tant dans les espaces intermicrovilleux que dans le cytoplasme de la cellule épithéliale et leur acheminement à travers ce dernier est bien distinct de celui des autres triglycérides homogènes expérimentés.

\section{Acides gras}

Comme les triglycérides, les acides gras (tabl. 3 ) sont absorbés dès le début du duodénum et leur absorption ne s'arrête, dans les conditions d'expérience, que $30 \mathrm{~cm}$ environ avant la valvule de Bauhin. Dès le commencement du duodénum, l'acide linoléique et 1'acide oléique sont absorbés avec une intensité apparente moyenne et légèrement supérieure à celle de l'acide laurique; quant à l'acide palmitique et à l'acide stéarique, l'absorption en est nettement moins intense et ces observations sont valables pour toute la longueur de l'intestin (fig. 3). Pour ces deux derniers acides, bien qu'ils se colorent avec moins d'intensité que les autres, même à leur température de fusion, il semble bien qu'ils se trouvent régulièrement en quantité moins abondante à l'intérieur de la cellule absorbante, tandis que leur concentration dans le contenu intestinal reste constamment plus élevée. D'une façon générale, il semble 


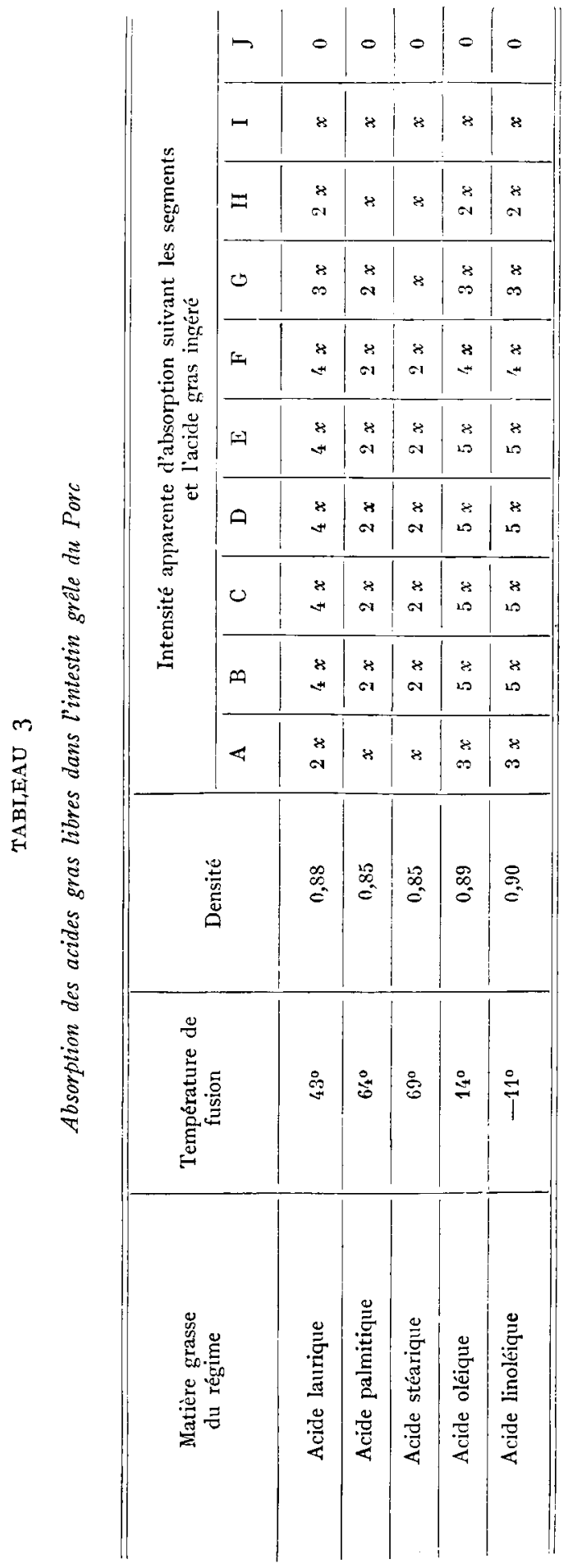


que les acides gras aient une intensité d'absorption apparente inférieure à celle des triglycérides correspondants.

Pour l'ensemble des acides gras, la surface absorbante est comparable ou légèrement inférieure à celle des triglycérides homogènes.

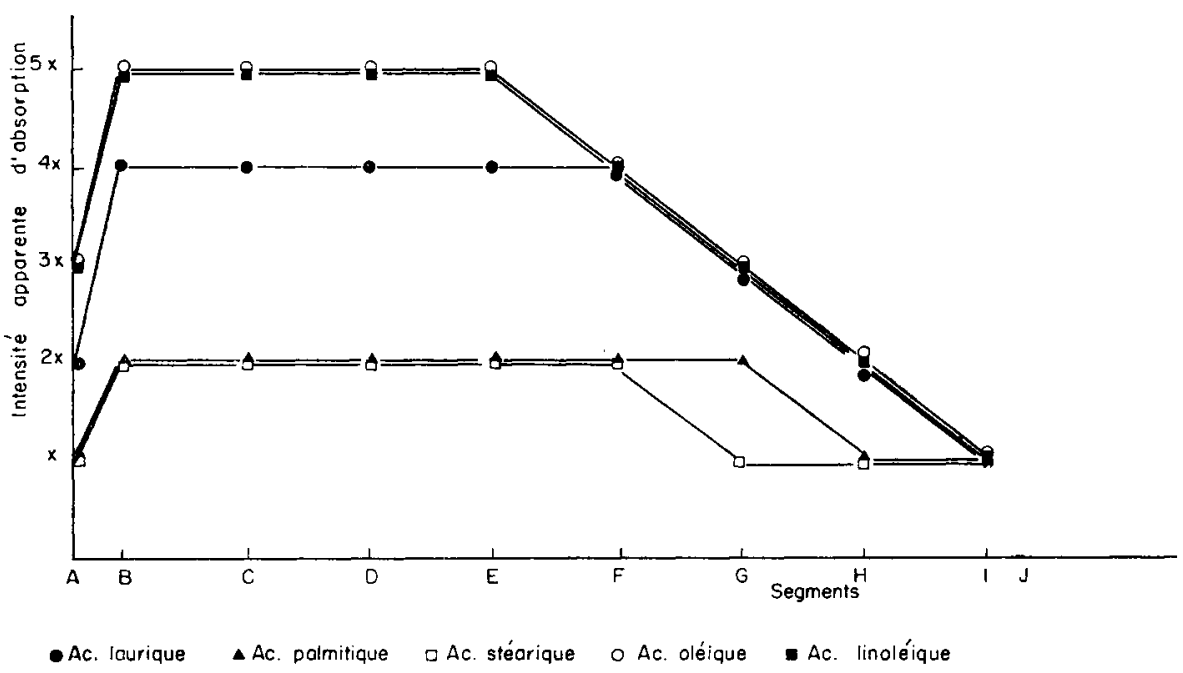

FIG. 3. - Absorption des acides gras libres dans l'intestin grêle du Porc

L,es particules soudanophiles, que l'on trouve dans les espaces intermicrovilleux et à l'intérieur des cellules, présentent un aspect peu différent, tout en étant caractéristique de chaque acide (fig. 8, 9).

Pour les trois séries d'expériences, l'intestin des animaux-témoins n'ayant pas reçu de graisses les dernières 48 heures avant le sacrifice a été examiné et, dans aucun des cas, il n'a été vu de matières soudanophiles dans les cellules de l'épithélium intestinal.

\section{DISCUSSION ET CONCLUSION}

Les résultats obtenus dans les conditions d'expérience montrent que les graisses de nature différente commencent à être absorbées au début du duodénum (dès les premiers centimètres après le pylore). Le début de l'absorption, dont l'intensité apparente varie avec la nature de la graisse, est généralement faible pour chaque cellule absorbante ; la surface d'absorption est presque toujours localisée au sommet des villosités. Il est intéressant, toutefois, de constater que l'absorption commence, tant pour les glycérides que pour les acides gras, Io à $I 5 \mathrm{~cm}$ avant le débouché du canal pancréatique dans le duodénum. Pour ce qui est des triglycérides, il faut admettre qu'ils ont été partiellement hydrolysés, soit avant l'ingestion, soit dans l'estomac, ou qu'il existe un reflux du suc pancréatique en direction du pylore, la présence d'acides gras ayant été démontrée par la coloration spécifique au Bleu de Nil. 
C'est dans la partie distale du duodénum et dans les deux premiers tiers du jéjunum-iléon que l'absorption est la plus forte pour chacune des graisses expérimentées, tant en ce qui concerne son intensité apparente qu'en ce qui concerne l'étendue de la surface absorbante.

Les expériences de BorGSTRöm et al. (I957, I962), qui montrent que l'huile de maïs, dans un repas équilibré, est absorbée, chez l'Homme, dans la partie distale du duodénum et au début du jéjunum sont confirmées par les nôtres, chez le Porc, à ceci près, que, dans nos expériences, les repas étant surchargés en graisses, l'absorption continue plus loin.

Pour Frazer (I943), chez le Rat, l'huile d'olive et l'acide oléique sont absorbés sur le même tronçon du jéjunum proximal: nos observations sont concordantes en ce qui concerne l'absorption de ces substances sur la même partie de l'intestin, mais, probablement à cause de la composition différente du repas, l'absorption, chez le Porc, commence déjà dans le duodénum. Il en est de même pour les expériences de Johnstor (I959) avec l'acide palmitique.

La constatation faite, par BENSON (I956), CHANDLER et BENSON (I956), à savoir que l'huile d'olive administrée seule est absorbée, chez le Rat, dans la partie distale de l'intestin grêle, celle faite par ABERDEEN, SHEPHERd et Simmonds (I960), confirmée par BenNetT et Simmonds (I962), à savoir que l'huile de coprah l'est également à cet endroit, ainsi que l'expérience de FAVARGER (I949), sur le Chien, avec la triélaïdine, nous ont incité à faire ingérer au Porc, en marge de nos expériences, roo $\mathrm{g}$ d'huile de mais, sans autre aliment, pour déterminer si l'absorption se ferait de la même façon. Dans ces conditions, probablement à cause d'une propulsion stomacale plus forte du fait du changement dans la nature et le volume du repas, l'absorption commence non plus au début du duodénum, mais au niveau du deuxième quart du jéjunum-iléon et se poursuit sur le reste de l'intestin d'une façon très intense et quasi uniforme. Cette dernière observation permet de penser que les résultats obtenus par les auteurs précédents ne démontrent pas que la partie distale de l'intestin grêle est un lieu d'absorption préférentiel pour ces trois substances, mais que, dans les conditions d'expérience, l'absorption n'a pu se faire dans la partie proximale. D'ailleurs, comme nous l'avons dit plus haut, ABERDEEN a signalé que, si l'on supprimait la propulsion exercée par l'estomac en déposant le repas dans le dudoénum, l'absorption se faisait bien avant.

Les résultats contradictoires obtenus sur le lieu d'absorption de la trioléine et de l'acide oléique par Turner (I958) d'une part, par Grenier et al. (I962, I963), d'autre part, s'expliquent par la divergence de leurs méthodes expérimentales.

Dans nos expériences, où la quantité de graisse incorporée au repas est administrée en surcharge, c'est-à-dire en quantité supérieure à la capacité absorbante de l'intestin, l'absorption continue dans la troisième partie de l'intestin grêle jusqu'à $30 \mathrm{~cm}$ environ - très approximativement - avant la valvule de Bauhin, avec une intensité apparente, ainsi qu'une surface d'absorption, moins importante pour chacun des corps gras que dans le reste de l'intestin.

En somme, si on compare les lieux d'absorption suivant les segments correspondants, entre le pylore et la valvule de Bauhin, pour les différentes graisses ingérées séparément et administrées d'une façon physiologiquement normale, on peut conclure qu'il n'existe pas de segments de l'intestin de Porc, où une graisse soit absorbée d'une façon préférentielle par rapport à une autre. En outre, on constate que, d'une 
part, l'intensité d'absorption, pour l'ensemble des graisses expérimentées, varie suivant les segments et que, d'autre part, les graisses de différente nature chimique sont absorbées avec une intensité différente, sur toute la longueur de l'intestin où se fait l'absorption.

En ce qui concerne l'intensité d'absorption par segments, elle semble être fonction de l'état physico-chimique des graisses ingérées, de leur concentration et de la vitesse de transit du bol alimentaire ; ce dernier semble être conditionné par la quantité de nutriment ingéré, par sa composition qualitative et quantitative et par la façon dont il est administré.

Ainsi, dans la partie proximale du duodénum où, d’une part, la vitesse de transit est accélérée par la propulsion pylorique, où d'autre part, la transformation physicochimique des graisses sous l'action des sels biliaires (dont 1'importance a été mise en évidence par BERNARD (I856), plus récemment par FRÖLICHER (I936) et confirmée depuis par d'autres chercheurs) et du suc pancréatique ne fait que commencer, l'absorption, sans être identique pour toutes les graisses, est toujours faible.

Dans le cas de nos expériences, la partie distale du duodénum et les deux tiers proximaux du jéjunum-iléon sont la partie de l'intestin ò̀ l'intensité d'absorption est la plus forte et la surface d'absorption la plus grande pour l'ensemble des graisses expérimentées. On peut donc penser que c'est dans cette partie de l'intestin grêle que des facteurs tels que vitesse de transit, concentration et état physico-chimique des graisses ingérées sont optima, sans toutefois que le pouvoir réel d'absorption de la cellule épithéliale absorbante soit en cause.

Pour la partie distale du jujénum-iléon, l'intensité de l'absorption, ainsi que l'étendue de la surface absorbante, deviennent de moins en moins importantes et sont différentes suivant la nature des graisses ingérées. Cette diminution de l'absorption ne peut être, à notre avis, considérée comme un signe de diminution du pouvoir absorbant de la cellulle épithéliale, mais elle est la conséquence de l'affaiblissement de la concentration en graisses et de l'augmentation de la densité du contenu intestinal (retenu par la valvule de Bauhin), qui limitent le contact entre les particules de graisses et la surface absorbante. Ceci trouve sa confirmation dans l'expérience citée plus haut, qui montre que 1'intensité de l'absorption et la surface absorbante sont très grandes dans la partie distale de l'intestin, lorsque l'on fait ingérer de l'huile de maïs en quantité importante et sans autre aliment.

Pour ce qui est de l'inégalité de l'absorption des graisses de nature chimique différente, DEUEL, et al., l'avaient déjà constatée (I940).

Dans la série des acides gras expérimentés, l'acide linoléique et l'acide oléique, insaturés à un degré différent, puis l'acide laurique à longueur de chaîne moyenne, sont absorbés régulièrement avec une intensité très supérieure à celle qui caractérise l'absorption de l'acide palmitique et de l'acide stéarique, dont la chaîne est longue et saturée, ce qui confirme les résultats de FAVARGER et de GERLACH (I953), d'une part, et ceux de BENNETT (I964), d'autre part, parmi d'autres.

Ces observations sur les acides gras peuvent être valablement appliquées aux triglycérides homogènes expérimentés : ainsi la capacité d'absorption de l'intestin ris-à-vis de la tributyrine est plus importante que vis-à-vis de la trilaurine ou de la trioléine et l'intensité apparente d'absorption de ces dernières est bien supérieure à celle de la tristéarine et de la triplamitine. Cependant, d'une façon générale, les acides gras libres, ingérés séparément, semblent avoir un acheminement vers le 
" stroma " à travers le cytoplasme de la cellule épithéliale absorbante plus lent que lorsqu'ils sont ingérés sous forme de triglycérides homogènes correspondants.

L'estérification au niveau de la cellule épithéliale ne serait-elle pas plus lente dans le premier cas? C'est ce qui permettrait d'expliquer nos observations, ainsi que celles de Reiser (I950), Borgström (I952), Malm, ReEmTsma et Barke, (I956) et Favarger et GerLach (I953).

En ce qui concerne les triglycérides naturels expérimentés, l'intensité d'absorption de chacun d'eux est supérieure à celles des acides dont ils sont formés, si ces derniers sont ingérés libres et séparément.

Dans l'état actuel de nos recherches, il n'est pas possible de donner une interprétation quant à l'aspect des particules observées à l'intérieur du cytoplasme de la cellule épithéliale absorbante et qui diffère suivant la nature des graisses ingérées.

Reçu pour publication en décembre 1964 .

\section{REMERCIEMEN'S}

Ce travail a été effectué avec l'aide financière de la Délégation générale à la Recherche scientifique et technique (Comité de nutrition animale et humaine).

\section{SUMMARY}

\section{SITE OF ABSORPTION OF FAT IN THE SMALL INTESTINE OF THE PIG}

A feed of $500 \mathrm{~g}$, balanced for protein and carbohydrate, but witch a high proportion of fat, was given to 3 groups of 7 pigs of 50 to $60 \mathrm{~kg}$ liveweight.

For the first group the fat, a total of $100 \mathrm{~g}$, consisted of a natural triglyceride supplied as an

il or fat, for the second group it was a homogeneous synthetic triglyceride and for the third it was given as a fatty acid.

The pigs were killed $6 \mathrm{I} / 2 \mathrm{~h}$ after the feed has been given. $\Lambda t$ that time the whole length of the small intestine is involved in the process of absorption. The large amount of the test fat given ensured that there was a sufficient amount to allow absorption, which depends on its concentration, to take place.

In all the animals, including controls, sections of the wall of the intestine were then examined histologically. Ten sections between the pylorus and the valvule of Bauhin werc studied.

It was shown that for all the fats tested absorption began immediately after the pylorus. The intensity of absorption, measured with a cytophotometer and termed apparent intensity, was small at that point. That could be explained by the high rate of passage of the bolus of feed. The intensity of absorption also differed according to the nature of the fat, which could be due to the particular physico-chemical state of each one.

Absorption took place along the whole length of the duodenum and the jejunum-ileum up to about $30 \mathrm{~cm}$ before the valvule of Bauhin. That was true for all the substances given except tributyrine which disappeared very early from the contents of the intestine. This would suggest that the absorptive capacity of the intestine is greater for this substance than for the others.

Intensity of absorption was greatest at the end of the duodenum and the proximal two thirds of the jujenum-ileum. In the last third of the jujenum-ileum it was less because of the reduced concentration of fat in the contents of the intestine and the accumulation of the contents.

As regards the absorbing surface, at the start of the duodenum it was limited to the tips of the villosities. In the distal part of the duodenum and the proximal two thirds of the jejunumileum on average two thirds of the villosity were involved, and in the distal part of the jejunum-ileum the absorptive surface was irregular and less extensive.

In conclusion it may be said that in the conditions of these experiments there was no preferred site for absorption of the different fats, which were all absorbed along the whole length of the small intestine. 


\section{RÉFÉRENCES BIBI,IOGRAPHIQUES}

Aberdeen V., Shepherd P. A., Simmonds W. J., 196o. Concurrent measurement, in unanaesthetized rats, of intestinal transport and fat absorption from the lumen. Quart. J. exper. Physiol., 45, 265-274.

BennetT S., Simmonds W. J., r962. Absorptive capacity and intestinal motility in unanaesthetized rats during intraduodenal infusion of fat. Quart. J. exper. Physiol., 49, $210-216$.

BennetT S., I964. Intestinal absorptive capacity and site of absorption of fat under steady state conditions in the unanaesthetized rat. Quart. J. exper. Physiol., 49, 2 10-218.

Benson J. A., Chandler G. N., Vansteenhuyse F. E., Gagnon J. O., 1956. Studies concerning the site of fat absorption in the small intestine of the rat. Gastroenterology, 30, 54-6r.

BERNARD C., 1856. Mémoire sur le pancréas et sur le rôle du suc pancréatique dans les phénomènes digestifs, particulièrement dans la digestion des matières grasses neutres. Baillière, Paris.

Bernard K., Wagner H., Ritzel G., I952. Versuche zur quantitativen Erfassung der bei der Resorption von Neutralfett eintretenden Spaltung. Helv. Chim. Acla., 35, 1404-14II.

Borgstrom B., I952. On the mechanism of the intestinal fat absorption. IV. Metabolism of lipids. 6 . Acta physiol. scand., 25, 291-314.

Borgstrom B., Dahlqvist A., Lundh G., Sjövall J., I957. Studies of intestinal digestion and absorption in the human. J. clin. Invest., 36, $1521-1536$.

Borgstrom B., Dahlovist A., Lundh G., I962. On the site of absorption of fat from the human small intestine. Gut, 3, 3I5-3I7.

Chandler G. N., Benson J. A., 1956. The site of fat absorption in the rat. Gastroenterologia, 85, I60-168.

Deuel H. J. jr., Hallman L., Leonard A., 1940. The comparative rate of absorption of some natural fats. J. Nutr., 20, 2 I 5-226.

Favarger P., 1949. La participation des phospholipides à la résorption des graisses dans les différentes régions de l'intestin de chien. Helv. physiol. pharm. Acta, $7,37 \mathrm{I}-38 \mathrm{r}$.

Favarger P., Gerlach J., I953. Variations dans le mode de résorption des différentes graisses et acides gras chez le rat. Hel. physiol. pham. Acta, 11, 239-250.

Frazer A. C., 1943. Differenciation in the absorption of olive oil and oleic acid in the rat. J. Physiol., 102, 306-3 I 2 .

Prolicher E., I936. Die Resorption von Gallensaüren aus verschiedenen Dünndarmabschnitten. Biochem. Z., 283, 273-279.

Grenier J. F., Hatano M., Weiss A. G., ig62. Sièges électifs au niveau de l'intestin grêle de l'absorption de la trioléine ${ }^{14} \mathrm{C}$ et de l'acide oléique ${ }^{14} \mathrm{C}$. C. R. Soc. biol., 156, I695-1697.

Grenier J. F., Hatano H., Sava G., Crevoisier R., 1963. Sièges électifs de l'absorption de la trioléine ${ }^{14} \mathrm{C}$ et de l'acide oléique ${ }^{-14} \mathrm{C}$ au niveau de l'intestin. Rôle respectif de la muqueuse intestinale, de la bile et du suc pancréatique. C. R. Soc. biol., 15\%, 1502-1 505.

JensEnius H., I945. Results of experimental resections of the small intestine of dogs. Universitetsforlager NYT Nordisk Forlog, Copenhagen, Arnold Busck.

Johnston J. M., 1959. Site of fatty acid absorption. Proc. Soc. exper. Biol. Med., 100, 669-670.

Knoebel L. K., Nasset L. S., 1957. The digestion and absorption of fat in dog and man. J. Nutr., 61, 405-4I9.

Kremen A. J., LiNNer J. H., Nelson H. G., 1954. An experimental evaluation of the nutritional importance of proximal and distal small intestine. Ann. Surg., 140, 439-447.

Malm J. R., Reemtsma K., Barker H. G., 1956. Comparative fat and fatty acid intestinal absorption test utilizing radioiodure labelling. Results in normal subjects. Proc. Soc. exper. Biol. Med., 92, 47 I-474.

McNaUght A. B., I959. The comparative histology of fat absorption : a review of past work with original observations. Scot. med. J., 4, I I-26.

Razumov M. I. rg63, Intestinal villi cells and fat assimilation. Fed. Proc, 22, Part ri. T 31г. T 31 I.

REISER R. 1950, Conjugated linoleic acid in rat tissue lipids after ingestion as free acid and as triglyceride. Proc. Soc. exper. Biol. Med., 74, 666-669.

Turner D. A. $195^{8}$, The absorption, transport, and deposition of fat. Application of a new method for the determination of $1^{131}$-lipid activity in dogs and man. a et b. Amer. J. Digest. Dis., 3, 594-640, 682-708.

Vodovar N., lílanzy J., Françors A. C. I964, Intestin grêle du Porc. I. Dimensions en fonction de l'âge et du poids, étude de la jonction du canal cholédoque et du canal pancréatique à celui-ci. Ann. Biol. anim. Bioch. Biophys., 4, 27-34

Vodovar N. I964, Intestin grêle du Porc. II. Structure histologipue des parois et plus particulièrement de la tunique muqueuse en fonction de l'âge de l'animal. Ann. Biol, anim. Bioch, Biophys., 4, i I $3^{-1} 39^{\text {. }}$ 
Firg. 4 à 9. - Aspect général de l'acheminement de différentes matières grasses à travers les espaces intermicrovilleux et le cyloplasme de la cellute épilhéliale absorbante du Porc

FIG. 4. - Aspect des particules d'huile de coprah au cours de leur acheminement à travers le cytoplasme dans le premier tiers du péfumum-iléon. $\times$ I 500

Fuc. 5. - Aspect des particules de saindoux au cours de leur acheminement à travers le cytoplasme dans le premier tiers du jéjunum-iléon. $x$ I 500

Fig. 6. - Aspect des particules de trilatrine an tond des espaces intermicrovilleux et dans le cytoplasme (fin du duolénum). $\times$ I 500

FIG. 7. - Aspect des particules de tristéarine au fond des espuces intermicrovilleux et dans le cytoplasme ( $f$ in du duodénum). $\times$ I 500

FIG. 8. - Aspect de l'absorption de l'acide oléique dans la partie proximale du jéjunum-iléon. $\times$ I 500

FIG. 9. - Aspect de l'absorption de l'acide laurique dans la partie proximale du jéjunum-iléon. $x$ I 500 

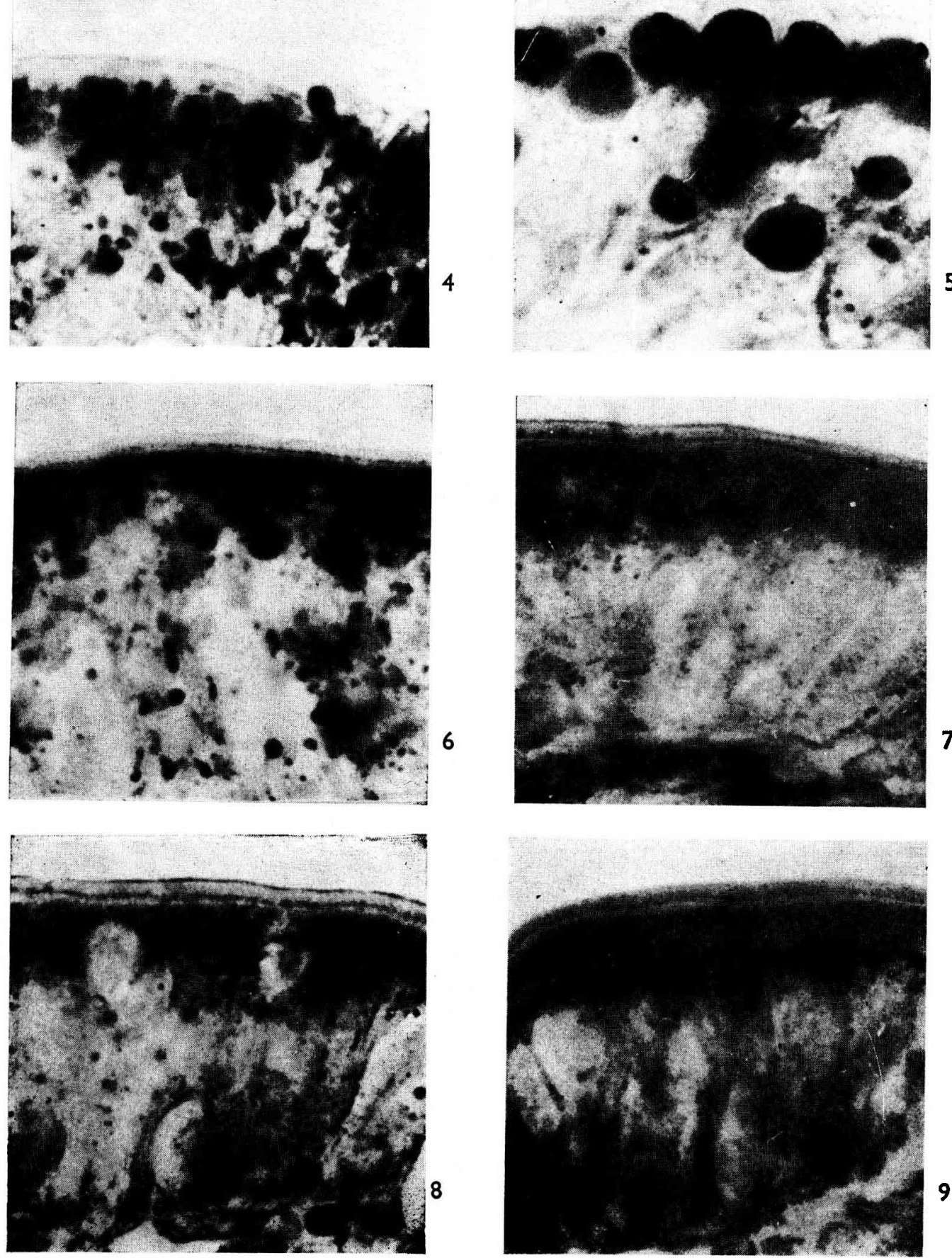